\title{
Back to the future? The role of temporal focus for mapping time onto space
}

\begin{tabular}{|r|l|}
\hline Journal: & Quarterly Journal of Experimental Psychology \\
\hline Manuscript ID & QJE-STD-18-122.R2 \\
\hline Manuscript Type: & Standard Article \\
\hline Date Submitted by the & 25 -Jun-2019 \\
\hline Complete List of Authors: & $\begin{array}{l}\text { Bylund, Emanuel; Stellenbosch University, General Linguistics } \\
\text { Gygax, Pascal; University of Fribourg, Department of Psychology } \\
\text { Samuel, Steven; Stockholm University, Centre for Research on } \\
\text { Bilingualism; University of Essex } \\
\text { Athanasopoulos , Panos ; Lancaster University, Department of } \\
\text { Linguistics; Stellenbosch University }\end{array}$ \\
\hline Keywords: & temporal focus, conceptual metaphor, cross-cultural, space, time \\
\hline &
\end{tabular}

\section{SCHOLARONE" \\ Manuscripts}


Title:

Back to the future? The role of temporal focus for mapping time onto space

\author{
Word count: \\ 5952
}

Authors:

Emanuel Bylund 1,2

Pascal Gygax ${ }^{3}$

Steven Samuel 2, 4

Panos Athanasopoulos 5,1

1) Stellenbosch University, South Africa

2) Stockholm University, Sweden

3) University of Fribourg, Switzerland

4) University of Essex, UK

5) Lancaster University, UK

Corresponding author:

Emanuel Bylund

mbylund@sun.ac.za

Department of General Linguistics

Stellenbosch University

7600 Stellenbosch

South Africa 


\title{
Back to the future? The role of temporal focus for mapping time onto space
}

\begin{abstract}
Do we conceptualise the future as being behind us or in front of us? While this question has traditionally been investigated through the lens of spatiotemporal metaphors, new impetus was recently provided by the Temporal-Focus Hypothesis (de la Fuente et al., 2014, Psych Sci). This hypothesis holds that the mapping of temporal concepts onto the front-back axis is determined by an individual's temporal focus, which varies as a function of culture, age, and short-term attention shifts. Here, we instead show that participants map the future on to a frontal position, regardless of cultural background and short-term shifts. However, one factor that does influence temporal mappings is age, such that older participants are more likely to map the future as behind than younger participants. These findings suggest that aging may be a major determinant of space-time mappings, and that additional data need to be collected before concluding that culture or short-term attention do influence space-time mappings.
\end{abstract}

Keywords: temporal focus; conceptual metaphor; cross-cultural; space; time, gesture

Humans have a prepotent tendency to map concepts of time onto spatial frames. Not only do we often talk about the future as being in front of us (Evans, 2013; Haspelmath, 1997), but we tend to think about it in the same way (e.g., Boroditsky, 2000; Miles, Nind, \& Macrae, 2010; Torralbo, Santiago, \& Lupiáñez, 2006). A possible explanation for these conceptualisations may be found in our interactions with the physical world: as we move through space, the distance we have passed lies behind us, and the distance yet to be covered lies ahead of us (Clark, 1973; Radden \& Dirven, 2007). However, while this perceptuo-motor experience is arguably universal, some languages and some cultural groups nonetheless conceptualise future events as behind, and past events as in front (e.g., Dahl, 1995; de la Fuente, Santiago, Roman, Dumitrache, \& Casasanto, 2014; Núñez \& Sweetser, 2006) - if using a sagittal axis at all (Boroditsky, 2001; Boroditsky \& Gaby, 2010; Núñez, Cooperrider, Doan, \& Wassmann, 2012). The embodied experience of moving through time and space thus fails to account for this variation.

A radically different possibility, recently captured in the Temporal-Focus Hypothesis (de la Fuente et al., 2014), is that the mapping of time onto spatial frames is ultimately determined by how much attention you devote to the past and the future. The underlying assumption here is that our focus of attention is literally in front of our eyes. In a series of experiments, de la Fuente and associates 
showed that thinking about the future renders you more likely to conceptualise it as in front and the past as behind, whereas devoting attention to the past yields the reverse pattern.

By introducing temporal focus as a determinant of the mapping of time onto space, the Temporal-Focus Hypothesis directs the spotlight on a concept that hitherto has been used in economics, health, and memory research (e.g., Guo, Ji, Spina, \& Zhang, 2012; Ji, Guo, Zhang, \& Messervey, 2009; McKay, Percy, Goudie, Sumnall, \& Cole, 2012; Shipp, Edwards, \& Lambert, 2009). An accumulating number of studies now suggest that temporal focus may successfully account for the spatialisation of time at the three crucial levels of analysis identified by Núñez and Cooperrider (2013): At the highest level, temporal focus seems to explain cross-cultural variation in space-time associations, as seen in correlations reported between culture-specific temporal attitudes and frontback mappings (de la Fuente et al., 2014; Li, Bui, \& Cao, 2018). At the intermediate level of analysis, temporal focus seems to account for individual variation within cultures, as seen in differences in mappings associated with pregnancy, age, and circadian rhythm (de la Fuente et al., 2014; Li, 2018; Li $\&$ Cao, 2018b). Finally, at the finest level of analysis, that of moment-to-moment variability within individuals, changes induced in temporal focus have been reported to trigger corresponding changes in front-back mappings (de la Fuente et al., 2014; Li \& Cao, 2018a).

The current paper replicates de la Fuente et al.'s (2014) methodology along the three levels of analysis proposed by Núñez and Cooperrider (2013) with the following new populations: at the highest level, we compare British and Afrikaner participants' temporal focus; at the intermediate level we test young and old Afrikaner participants; and at the finest level, we test British, Afrikaner, and Swiss French participants. Finally, we also run a series of meta-analyses of past and current findings, as a more stringent test of the effects of temporal focus on temporal cognition to date, allowing us to discuss the currently available evidence in context, and thus propose several avenues for future research.

Experiment 1. Do cross-cultural differences in temporal focus, but not in gesture, yield contrasting space-time mappings?

To test whether cultural differences in temporal focus yields differences in temporal thought, we assessed temporal focus and front-back mappings in individuals with Afrikaner (South Africa) and British English (UK) cultural background. British English culture is generally described as being future-oriented, whereas Afrikaner culture is commonly characterised as more traditional and conservative (Brook Napier, 2007; Giliomee, 2011; Johnston, 2014), and therefore, more pastoriented. According to the predictions of the Temporal-Focus Hypothesis, Afrikaners should map the past as in front, because they devote substantial attention to it (similar to the Moroccan Darija tested by de la Fuente et al., 2014). The English, in contrast, should have their temporal focus set on the future and thus conceptualise it as in front (as did the Spaniards in de la Fuente et al.'s experiments). 
To test the robustness of the effects of cultural temporal focus on space-time mappings, we introduce a crucial difference to previous research: while Afrikaners may be past-oriented, they do not gesturally place the past in front of them, but behind them (see Pre-experimental study below). In other words, if Afrikaners exhibit greater past-focus than the British, then they should also map past events as in front, no matter how they gesture about them. ${ }^{1}$ However, if Afrikaners do not predominantly map past events as in front but nonetheless exhibit greater past-focus than the British, then temporal focus would appear to be guided by different mechanisms.

\section{Method}

\section{Participants}

Participants were 70 Afrikaners $\left(\mathrm{M}_{\text {age }}=21.2\right.$ years $)$ and 70 British $\left(\mathrm{M}_{\text {age }}=20.8\right.$ years $)$, all University students in South Africa and the UK, respectively.

\section{Materials and procedure}

A temporal diagram task, originally introduced by Casasanto (2009) and adapted by de la Fuente et al. (2014) was used to assess participants' front-back mappings. The diagram showed a cartoon character seen from above, with one box in front and one box behind him (Figure 1). Participants read that the character ("John" in English and "Jan" in Afrikaans) had visited a friend yesterday who liked plants, and would visit a friend tomorrow who likes animals, and were told to mark which box belonged to the past and future visits with $\mathrm{A}$ (for animals) and $\mathrm{P}$ (for plants). The order of yesterday/tomorrow and plants/animals was counterbalanced.

Participants' temporal focus was measured with a questionnaire developed by de la Fuente et al. (2014). Participants indicated on a 5-point-scale their dis-/agreement with statements regarding the past (e.g. "Traditions and old customs are very important for me") and the future (e.g. "Traditions are not useful to the present and future society").

As in de la Fuente et al. (2014), participants first performed the temporal diagram task and then answered the questionnaire.

\section{Design}

In this and subsequent experiments, we have aimed to obtain comparable sample sizes to previous research. More importantly though, we report Bayes factors to assess the relative strength of our evidence, which is particularly useful in determining whether non-significant results are due to data insensitivity (e.g., lack of statistical power) or true support of the null hypothesis over the alternative

\footnotetext{
${ }^{1}$ Note that the link between gestural and other kinds of behavioural space-time mappings need not be straightforward: In a study by Román, Santiago, Jasmin and Casasanto (2014, cited by de la Fuente et al., 2014), Spanish speakers showed only "a weak tendency" (p. 1683) to gesturally place future events in front, yet on a temporal diagram task exhibited an overwhelming preference (88\%) for future-in-front mapping.
} 
hypothesis (in this case, the Temporal-Focus Hypothesis) (Dienes, 2014, 2016). Following standard procedure, Bayes factors were calculated using 0 as lower bound, and (converted) effect sizes reported in previous research as rough estimates of the upper bound (see Supplemental Materials Online, SOM 1). Following Jeffreys (1961), Bayes factors less than $1 / 3$ were interpreted as substantial evidence of the null hypothesis, and factors greater than 3 as substantial evidence for the Temporal-Focus Hypothesis.

\section{Pre-experimental study: Temporal gestures in Afrikaans}

To examine Afrikaners' typical temporal gestures, deliberate temporal gestures were elicited from a total of 40 Afrikaners (university students residing in South Africa, with an average age of 21.1 years). The method used was the gesture elicitation paradigm established by Casasanto and Jasmin (2012), which contains a set of questions starting with "how would you gesture about something that..." followed by an imagined future or past event (see SOM). Participants were approached by a native Afrikaans speaker and asked to produce gestures according to the specific set of questions they were asked (translated into Afrikaans). Each participant answered two questions, one about the future, and one about the past. Their responses were recorded and analysed with regards to the spatial axis (e.g., lateral, sagittal) they used in their gestures. Seventy (or 87.5\%) out of the 80 elicited gestures made reference to a sagittal axis. Crucially, all of the sagittal gestures $(100 \%)$ adhered to the future-in-front and past-behind pattern, making reference to the front for future events and to the back for past events. A lateral axis was used in $11.25 \%(n=9)$ of the gestures: of these, $66.67 \%(n=6)$ placed the past to the left. One $(1.25 \%)$ gesture was produced referring to a vertical axis, indicating the future upwards.

\section{Results}

Temporal focus

A 2 (cultural group: Afrikaners vs. British) x 2 (temporal focus: past vs. future) mixed ANOVA of the groups' responses on the temporal focus questionnaire revealed a significant interaction, thus corroborating that the Afrikaners and the British differed in their temporal focus, $F(1,138)=28.15, p$ $<.001, \eta_{p}^{2}=.17$. A Bayes factor of $\mathrm{B}_{\mathrm{H}(0,0.99)}=4,57 \times 10^{5}$ indicated that the data was powerful enough to truly detect an interaction (i.e. $B>3$ ). Afrikaners showed greater agreement with past-focused statements than the British $(p<.01)$, who in turn showed greater agreement with future-focused statements than the Afrikaners $(p<.01)$ (Figure 1). This finding thus resonates with previous anthropological and sociological research, and lays a fruitful ground for comparing the space-time mappings of these groups.

[FIGURE 1]

[FIGURE 2] 


\section{Front-back mapping of time}

Contrary to what would be predicted on the basis of their divergent temporal foci, the Afrikaner and the British participants did not differ in their front-back mappings, Wald $\chi^{2}(1, N=140)=.05, p=.82$, Odds Ratio $=1.11,95 \% \mathrm{CI}=[.45,2.72]$ (Figure 2). Instead, both groups showed a strong preference for locating the future event in the front box (around 83\%). The Bayesian analysis shows that the data constitute substantial evidence of the null hypothesis over the Temporal-Focus Hypothesis, $\mathrm{B}_{\mathrm{H}(0,3.75)}=$ .15 (i.e. $\mathrm{B}<1 / 3$ ).

These results thus indicate that gestural behaviour that maps the future in front and the past behind may be dissociated from culture-specific attitudes towards the future and the past. On a more general level, this finding also raises questions as to the general robustness of the effects of cultural attitudes on space-time mappings. This issue is best approached by running a meta-analysis of the effects reported in previous studies, which produces a reliable indication of the overall strength of the effect of cultural attitudes on spatial mapping. For this purpose, we used the Exploratory Software for Confidence Intervals (Cumming, 2013) to run a random effects meta-analysis ${ }^{2}$ to integrate results from four experiments testing cross-cultural differences in front-back mapping (i.e., de la Fuente et al., 2014: Exp1; Gu et al., 2016: Exp1; Li et al., 2018, ${ }^{3}$ and this study: Exp1). The integrated effect size was medium to large, $d_{\text {unbiased }}=.71,95 \%$ CI $[.01,1.57]$, yet the CIs were indicative of a rather large heterogeneity of the effect. In other terms, whilst there is some evidence of cross-cultural differences, more data would need to be collected to examine this effect, as well as the source of its heterogeneity.

\section{Correlations between cultural temporal focus and front-back mappings}

To further assess the apparent dissociation between cultural temporal focus and spatial mappings, we followed de la Fuente et al. (2014) calculating a temporal focus index based on the participants' responses on the temporal focus questionnaire $\left(M_{\text {future-focused statements }}-M_{\text {past-focused }}\right.$ statements $) /\left(M_{\text {future-focused statements }}+M_{\text {past-focused statements }}\right)$, and then correlated this with performance on the temporal diagram. To maximise the likelihood of finding a significant relationship, correlations were run both across and within groups. However, temporal focus index did not successfully predict mapping preferences across groups, Wald $\chi^{2}(1, N=140)=.4, p=.53, \mathrm{OR}=.35,95 \% \mathrm{CI}=[.12$, 9.57], $\mathrm{B}_{\mathrm{H}(0,10.00)}=.11$, nor within groups: Afrikaners, Wald $\chi^{2}(1, N=70$, Afrikaners $)=.07, p=.79$, $\mathrm{OR}=.5,95 \% \mathrm{CI}=[.00,82.28], \mathrm{B}_{\mathrm{H}(0,10.00)}=.21$; the British, Wald $\chi^{2}(1, N=70)=.32, p=.57, \mathrm{OR}=$ $.23,95 \% \mathrm{CI}=[.00,39.56], \mathrm{B}_{\mathrm{H}(0,9.996)}=.17$.

\footnotetext{
${ }^{2}$ To obtain standardized Cohen's effect sizes from the odds ratios, we used the equation $d=L_{O R} \sqrt{3} / \pi$ (as advocated by Sánchez-Meca, Marín-Martínez, \& Chacón-Moscoso, 2003), where $\pi=3.14159$ and $L_{O R}$ is the natural logarithm of the odds ratio.

${ }^{3}$ Note that the authors tested cultural attitudes within a same culture/country in this study (i.e., North vs. South Vietnam)
} 
Again, this result provides substantial evidence for the null hypothesis over the Temporal-Focus Hypothesis, showing that also at the level of individuals, cultural attitudes towards the past and the future are not necessarily associated with the spatial mapping of these concepts. Although our results contradict those of de la Fuente et al. (2014), they can only be considered as cumulative, or complementary, in that they may well illustrate what Cumming (2014) coined as the dance of the CIs. In a nutshell, any sequence of an experiment replication will produce different $p$ values as well as different confidence intervals. To test this, the current result were entered into a random-effects metaanalysis together with previous findings on the temporal focus index (i.e., de la Fuente et al., 2014: Exp4; Li et al., 2018; ${ }^{4}$ Li \& Cao, 2018b: Exp1; and our study: Exp1), although the questionnaire to measure temporal focus was not always the same. The Exploratory Software for Confidence Intervals (Cumming, 2013) yielded an integrated effect size that was medium to large, $r=.58,{ }^{5} 95 \% \mathrm{CI}[.29$, .86], yet, the CIs were indicative of a rather large heterogeneity of the effect. Again, whilst there is some evidence for the Temporal-Focus Hypothesis, our data seem to suggest this evidence to be not entirely conclusive.

\section{Study 2: Does age influence space-time mappings?}

Next, we tested whether individual differences could predict front-back mappings of time. The individual factor we examined was age. Based on the insight that older people primarily rely on past achievements in their self-perceptions and dedicate more time on reminiscing the past (Butler, 1963; Wong \& Watt, 1991), along with the finding that the direction of space-time mappings systematically varies as a function of age (de la Fuente et al., 2014), we expected this effect to also obtain in a novel population, Afrikaners residing in South Africa, such that older individuals would be more likely to place past events in front than younger individuals.

\section{Method}

\section{Participants}

Sixty-one younger (University students, $\mathrm{M}_{\mathrm{age}}=20.4$ years) and 49 older (retirees, $\mathrm{M}_{\mathrm{age}}=74.9$ years) Afrikaners participated. The older participants were chosen against their performance on an Afrikaans version of the Mini Mental State Exam.

Materials, procedure, and design

In a direct replication of de la Fuente et al. (2014), front-back mappings were assessed with the temporal diagram of Experiment 1. See SOM 1 for statistical details.

\footnotetext{
${ }^{4}$ Note that the authors tested cultural attitudes within a same culture/country in this study (i.e., North vs. South Vietnam)

5 To obtain an approximation of Pearson's $r$, we used Cohen's $d$ and transformed it using the equation $r=\sqrt{\frac{d^{2}}{d^{2}+4}}$
} 


\section{[FIGURE 3]}

\section{Results}

Consistent with de la Fuente et al. (2014), the older group mapped past events in front significantly more often than the younger group ( $49 \%$ vs $20 \%)$, Wald $\chi^{2}(1, N=110)=10.07, p=.002$, OR $=3.92$, $95 \% \mathrm{CI}=[1.69,9.12]$ (Figure 3 ). With a Bayes factor of $\mathrm{B}_{\mathrm{H}(0,2.14)}=51.73$, this replicates the findings of de la Fuente et al. (2014) in a new population and provides considerable support for the TemporalFocus Hypothesis over the null hypothesis, thus suggesting that age may reliably account for individual variation in space-time mappings. We further ran a random effects meta-analysis to give us a better indication of the overall strength of the effect of age on space-time mapping, based on our data and that of de la Fuente et al. (2014) (Experiment 3). The integrated effect size was rather large, $d_{\text {unbiased }}=.97,95 \%$ CI $[.54,1.39]$, and the CIs were indicative of a high probability of the effect being present.

\section{Experiment 3. Does shifting the temporal focus reverse space-time mappings?}

While findings from previous studies (de la Fuente et al., 2014; Li \& Cao, 2018b) (and Experiment 2) show that space-time mappings may undergo changes during the life-span of an individual, we now turned to examine whether space-time mappings may also be flexibly altered on a much shorter timescale. Previous research has shown that priming and novel category learning may alter human time conception (Boroditsky, 2001; Casasanto \& Bottini, 2013; Miles, Tan, Noble, Lumsden, \& Macrae, 2011; Román, Flumini, Lizano, Escobar, \& Santiago, 2015). However, most of this research has relied on different kinds of spatial manipulations (e.g., spatial metaphors) of time representation, largely leaving aside the potential impact of attention/focus shifts. Ultimately, if the amount of attention devoted to the past or the future indeed determines front-back mappings, then shifting a person's temporal focus should yield corresponding differences in their mappings (de la Fuente et al., 2014). Experiment 3 tested this particular prediction of the Temporal-Focus Hypothesis.

\section{Experiment $3 a$}

\section{Participants}

Two-hundred and one Afrikaners (University students, $\mathrm{M}_{\mathrm{age}}=19.4$ years) took part in Experiment 3a.

\section{Material and Procedure}

De la Fuente et al.'s (2014) Temporal Induction Task was used to induce a shift in individual temporal focus. Participants were randomly allocated to either the past-induction condition $(N=101)$, where they had to write down answers to questions that forced them to focus on the past (e.g., "What made you feel afraid when you were little?"), or the future-induction condition $(N=100)$, with future-related questions (e.g., "What about the future makes you feel afraid?"). Once they had answered the 


\section{Method}

A total of 161 Swiss French $\left(\mathrm{M}_{\mathrm{age}}=20.6\right)$ and $104\left(\mathrm{M}_{\mathrm{age}}=21.3\right)$ British University students in Switzerland and the UK, respectively, were randomly allocated (within their respective group) to either the past-induction (Swiss sample: $N=87$; British sample: $N=52$ ) or the future-induction condition (Swiss sample: $N=77$; British sample: $N=52$ ). Materials, procedure and design followed Experiment 3a.

\section{[FIGURE 4A-C]}

\section{Results}

The Swiss French participants showed no effect of temporal induction in their performance on the temporal diagram, Wald $\chi^{2}(1, N=161)=.04, p=.845, \mathrm{OR}=.91,95 \% \mathrm{CI}=[.34,2.42], \mathrm{B}_{\mathrm{H}(0,2.78)}=$ 0.15. Instead, participants from both conditions showed a clear preference for future-in-front mappings (Figure 4b).

The British participants exhibited a similar degree of immutability in their mappings, with no significant difference between conditions, Wald $\chi^{2}(1, N=104)=.07, p=.791, \mathrm{OR}=1.15,95 \% \mathrm{CI}=$

\footnotetext{
${ }^{6}$ For instance, le future est devant nous ('the future is ahead of us'), le passé est derrière ('the past is behind') These linguistic patterns are however of little relevance here. The temporal induction task should yield a significant effect in the expected direction, irrespective of the conceptual metaphors of these languages.
} 
$[.41,3.26], \mathrm{B}_{\mathrm{H}(0,2.78)}=0.23$. Here too, participants clearly preferred future-in-front mappings (Figure $4 c)$.

For both the Swiss and British samples, the Bayes factors indicate substantial evidence for the null hypothesis over the Temporal-Focus Hypothesis, showing that the immutability of space-time mappings in young Afrikaners indeed generalises to other populations.

In all, for the three samples used in this experiment, we found no evidence of a successful induction of a shift in individual temporal focus. We further ran a random effects meta-analysis to give us a better indication of the overall strength of the shift in individual temporal focus effect, as primed by de la Fuente et al. (2014. For this purpose, we used the Exploratory Software for Confidence Intervals (Cumming, 2013) to run an initial random effects meta-analysis to integrate results across our three samples and that of de la Fuente et al. (2014). The integrated effect size was rather moderate, $d_{\text {unbiased }}=.36,95 \%$ CI $[-.42,1.14]$, and the CIs were indicative of a high heterogeneity of the effect. Essentially, these values indicate that a shift in temporal focus using the temporal induction priming technique may well be difficult to induce, although not impossible.

\section{General discussion}

In a series of experiments, we tested whether the concept of temporal focus can successfully predict implicit sagittal timelines at three levels of analysis. At the intermediate level - that of individual variation - the findings from Experiment 2 show that age is indeed a successful predictor of spacetime mappings, with older participants being more prone to place the past in front of them than younger participants. This is consistent with previous research on age-related temporal focus and orientation in general (e.g., Wong \& Watt, 1991), and space-time mappings in particular, replicating de la Fuente et al's (2014) findings from young and old Spaniards and Moroccan Arabic individuals in young and old Afrikaner individuals (2014), and corroborating this particular prediction of the Temporal-Focus Hypothesis.

However, at the highest level of analysis - that of cultural variation (Experiment 1) - substantial differences in the temporal focus of the groups under examination did not yield corresponding differences in space-time mappings. It thus seems that while temporal focus may reflect space-time mappings, as demonstrated by de la Fuente et al. (2014), such relationship may not be applicable to all cases. The fact that cultural differences in temporal focus were dissociated from temporal thinking in our samples questions the causal role of cultural temporal attitudes for spatial mapping.

At the finest level of analysis - that of moment-to-moment variability (Experiment 3) - the results show, across three independent populations, that future-in-front mappings are not altered by short-term shifts in temporal focus. In view of Casasanto's (2016) observation that space-time mappings in language are less likely to be flexibly modulated as linguistic conventions change slowly, it could be expected that temporal mappings driven by attention exhibit greater malleability, as 
attention is readily primed. However, the fact that no effect of temporal induction was found suggests that the attested front-back mappings are resistant to short-term shifts in temporal focus, thus questioning the power of temporal focus in priming space-time mappings (compared to, e.g., priming through spatial metaphors).

The series of meta-analyses that we conducted sheds considerable light on the potential underlying principles of the temporal focus hypothesis, but they also reveal an increasingly complex nexus of the interplay amongst culture, age, religion, and varieties of attention to time that is likely to be the focus of a prolonged series of future investigations. In Experiment 1, the meta-analyses showed that the effect of cultural background presents with a large heterogeneity. Differences in the ways in which different cultures assign value to the future or the past may well be idiosyncratic to the specific culture's historical and political characteristics. For example, Li et al. (2018) found that Southern Vietnamese were more future focused than Northern Vietnamese and attributed this to the assumption that the former place more value on economical and technological development while the latter tend to value more tradition. Our Afrikaner participants, like the Northern Vietnamese, tend to attach greater importance to tradition, but unlike the Northern Vietnamese, whose past focus seems to be intertwined with the current political regime that may value and promote conservative attitudes (Li et al., 2018), the Afrikaners live in a country with a troubled political history, associated with the apartheid regime that enforced racist policies of separation and oppression. For this reason, the past may carry implicit negative connotations that preclude any inclination by young Afrikaners to place it in front. However, this also has to be understood in the context of Afrikaner culture that places respect on elders, tradition, and the Church (Cloete, 1992; Giliomee, 2011; Johnston, 2014). This could explain the finding that our Afrikaner population in Experiment 1 are found to be past-focused when they have to consciously evaluate statements about the past and the future in the temporal focus questionnaire, but future focused in the more implicit measurement of temporal focus outside of any value judgment about the past or the future, such as that measured by the temporal diagram task. This phenomenon may be so pervasive that it may also account for the resistance of the same population to change their temporal focus in a rapidly-induced training context (Experiment 3). However, in Experiment 3 we also failed to replicate de la Fuente et al.'s (2014) rapidly induced changes in temporal focus in Spaniards to British and Swiss French participants. Indeed, the meta-analysis here suggests that a shift in temporal focus using the temporal induction priming technique may be very difficult to replicate. Cultural differences as to the connotative valence of the past may exist amongst Spaniards, Brits, and Swiss French, but we are unable presently to locate or articulate any possible locus of such differences that resides in those countries' historical and political past. The explanation here may lie on other cultural aspects or temporal experimental context. For example, $\mathrm{Li}$ and $\mathrm{Cao}$ (2018) found strong effects of religion on temporal focus (e.g., Taoists are more future-focused than Buddhists), and it is even the case that among Buddhists the extent of past or future focus can be modulated by a religion prime (Buddhists primed with Buddhas of the future are more future-focused than Buddhists primed 
with Buddhas of the past). It may well be the case that religious beliefs may affect the extent of the rapidly induced malleability of temporal focus, and one could attribute the lack of effect that we found in our populations to the fact that they are predominantly Christian, and therefore more future focused because of Christianity's emphasis on the second coming of Christ and the life ever after (this could be an additional factor on the Afrikaners participants' future in front predilection in the temporal diagram task - see previous paragraph). However, this would still fall short of explaining the presence of rapidly induced temporal focus changes in Spaniards in de la Fuente et al.'s (2014) study, who also come from a predominantly Christian background. Nevertheless, it is entirely possible that the degree of religious conviction/religiosity among Christians may play a role here, as it may be the case that our sample inadvertently included more religious Christians and/or fewer atheists, whereas de la Fuente et al.'s sample of Spaniards included more atheists and/or fewer religious Christians. Li and Cao (2018) found temporal focus shifts even in atheists when primed with religious prompts, but no study exists that has examined the effects of religiosity using the original temporal induction task by de la Fuente et al. (2014), also used in this study. Clearly, the interplay between religious belief and cultural background in shifting temporal focus certainly warrants further exploration. Finally, Li (2018) found that Chinese participants were more future focused if they were tested in the morning than in the afternoon. While this has not been examined using de la Fuente et al.'s (2014) temporal induction task, it opens up for the possibility that the time of day modulates the extent to which an individual may shift their attentional focus as a function of rapid training.

To conclude, while temporal focus may be associated with cultural attitudes towards time and with short-term attention shifts to different degrees and as a function of cultural particularities, it seems to hold great potential in predicting long-term shifts in implicit sagittal timelines. Conceptual representation is increasingly viewed as flexible and dynamic, shaped by a variety of experiential factors (e.g., language, culture, context; for overviews: Casasanto, 2016; Casasanto \& Lupyan, 2015). One factor that, to the best of our knowledge, is seldom included here is aging. While age itself has been assessed from a developmental perspective in the study of conceptual representation in children (e.g., Bowerman \& Levinson, 2001), the current findings from Experiment 2, in robustly replicating those of de la Fuente et al (2014), and the subsequent meta-analysis, show that age is a strong factor of temporal focus. A useful research tool that may immediately emerge from the currently available evidence is a biographical questionnaire used in conjunction with the temporal diagram task and all other relevant research tools (temporal focus questionnaire, temporal induction task) that captures not only age and cultural background, but also religious beliefs, political inclinations, attitudes towards cultural-historical past, parenthood and expectant parenthood, and gestural preferences for the past and the future. At present, it appears that the extent to which temporal focus is malleable is likely down to a combination of several factors, the exploration of which opens up a fruitful line of investigation into the temporal focus hypothesis in particular and into the wider issue of the cultural underpinnings of time conceptualization more generally. 


\section{Supplementary Material \\ The Supplementary Material is available at: qjep.sagepub.com}

\section{References}

Boroditsky, L. (2000). Metaphoric structuring: Understanding time through spatial metaphors. Cognition, 75(1), 1-28.

Boroditsky, L. (2001). Does language shape thought? Mandarin and English speakers' conceptions of time. Cognitive Psychology, 43(1), 1-22. https://doi.org/10.1006/cogp.2001.0748

Boroditsky, L., \& Gaby, A. (2010). Remembrances of Times East: Absolute Spatial Representations of Time in an Australian Aboriginal Community. Psychological Science, 21(11), 1635-1639. https://doi.org/10.1177/0956797610386621

Brook Napier, D. (2007). Languages, language learning, and nationalism in South Africa. In K. Schuster \& D. Witkosky (Eds.), Language of the Land: Policy, Politics, Identity (pp. 55-76). Charlotte, NC: Information Age Publishing.

Butler, R. N. (1963). The life review: An interpretation of reminiscence in the aged. Psychiatry, 26, 65-76.

Casasanto, D. (2009). Embodiment of abstract concepts: Good and bad in right- and lefthanders. Journal of Experimental Psychology. General, 138(3), 351-367. https://doi.org/10.1037/a0015854

Casasanto, D., \& Bottini, R. (2013). Mirror reading can reverse the flow of time. Journal of Experimental Psychology. General. https://doi.org/10.1037/a0033297

Casasanto, D., \& Jasmin, K. (2012). The hands of time: Temporal gestures in English speakers. Cognitive Linguistics, 23(4), 643-674. https://doi.org/10.1515/cog-20120020 
Clark, H. H. (1973). Space, time, semantics, and the child. In T. Moore (Ed.), Cognitive development and the acquisition of language (pp. 27-63). New York: Academic Press.

Cloete, E. (1992). Afrikaner Identity: Culture, Tradition and Gender. Agenda: Empowering Women for Gender Equity, (13), 42-56. https://doi.org/10.2307/4065612

Dahl, Ø. (1995). When the future comes from behind: Malagasy and other time concepts and some consequences for communication. International Journal of Intercultural Relations, 19(2), 197-209. https://doi.org/10.1016/0147-1767(95)00004-U

de la Fuente, J., Santiago, J., Roman, A., Dumitrache, C., \& Casasanto, D. (2014). When You Think About It, Your Past Is in Front of You: How Culture Shapes Spatial Conceptions of Time. Psychological Science, 25(9), 1682-1690. https://doi.org/10.1177/0956797614534695

Dienes, Z. (2014). Using Bayes to get the most out of non-significant results. Frontiers in Psychology, 5. https://doi.org/10.3389/fpsyg.2014.00781

Dienes, Z. (2016). How Bayes factors change scientific practice. Journal of Mathematical Psychology, 72, 78-89. https://doi.org/10.1016/j.jmp.2015.10.003

Evans, V. (2013). Language and time: A cognitive linguistics approach. Cambridge: Cambridge University Press.

Giliomee, H. (2011). The Afrikaners: Biography of a people. Cape Town: Tafelberg.

Guo, T., Ji, L.-J., Spina, R., \& Zhang, Z. (2012). Culture, temporal focus, and values of the past and the future. Personality \& Social Psychology Bulletin, 38(8), 1030-1040. https://doi.org/10.1177/0146167212443895

Haspelmath, M. (1997). From space to time: Temporal adverbials in the world's languages. LINCOM Europa.

Jeffreys, H. (1961). Theory of probability. Oxford, UK: Oxford University Press. 
Ji, L.-J., Guo, T., Zhang, Z., \& Messervey, D. (2009). Looking into the past: Cultural differences in perception and representation of past information. Journal of Personality and Social Psychology, 96(4), 761-769. https://doi.org/10.1037/a0014498

Johnston, A. (2014). South Africa: Inventing the nation. London: Bloomsbury.

Li, H. (2018). A future-minded lark in the morning: The influence of time-of-day and chronotype on metaphorical associations between space and time. Metaphor and Symbol, 33(1), 48-57. https://doi.org/10.1080/10926488.2018.1407995

Li, H., Bui, V. Q., \& Cao, Y. (2018). One country, two cultures: Implicit space-time mappings in Southern and Northern Vietnamese. European Journal of Social Psychology, 48(4), 560-565. https://doi.org/10.1002/ejsp.2356

Li, H., \& Cao, Y. (2018a). Karma or Immortality: Can Religion Influence Space-Time Mappings? Cognitive Science, 42(3), 1041-1056. https://doi.org/10.1111/cogs.12579

Li, H., \& Cao, Y. (2018b). The hope of the future: The experience of pregnancy influences women's implicit space-time mappings. The Journal of Social Psychology, 158(2), 152-156. https://doi.org/10.1080/00224545.2017.1297289

McKay, M. T., Percy, A., Goudie, A. J., Sumnall, H. R., \& Cole, J. C. (2012). The Temporal Focus Scale: Factor structure and association with alcohol use in a sample of Northern Irish school children. Journal of Adolescence, 35(5), 1361-1368. https://doi.org/10.1016/j.adolescence.2012.05.006

Miles, L. K., Nind, L. K., \& Macrae, C. N. (2010). Moving Through Time. Psychological Science, 21(2), 222-223. https://doi.org/10.1177/0956797609359333

Miles, L. K., Tan, L., Noble, G. D., Lumsden, J., \& Macrae, C. N. (2011). Can a mind have two time lines? Exploring space-time mapping in Mandarin and English speakers. Psychonomic Bulletin \& Review, 18(3), 598-604. https://doi.org/10.3758/s13423-0110068-y 
Núñez, R., Cooperrider, K., Doan, D., \& Wassmann, J. (2012). Contours of time: Topographic construals of past, present, and future in the Yupno valley of Papua New Guinea. Cognition, 124(1), 25-35. https://doi.org/10.1016/j.cognition.2012.03.007

Núñez, R., \& Sweetser, E. (2006). With the future behind them: Convergent evidence from Aymara language and gesture in the crosslinguistic comparison of spatial construals of time. Cognitive Science, 30(3), 401-450. https://doi.org/10.1207/s15516709cog0000_62

Radden, G., \& Dirven, R. (2007). Cognitive English Grammar. Amsterdam: John Benjamins Publishing.

Román, A., Flumini, A., Lizano, P., Escobar, M., \& Santiago, J. (2015). Reading direction causes spatial biases in mental model construction in language understanding. Scientific Reports, 5, 18248. https://doi.org/10.1038/srep18248

Shipp, A. J., Edwards, J. R., \& Lambert, L. S. (2009). Conceptualization and measurement of temporal focus: The subjective experience of the past, present, and future. Organizational Behavior and Human Decision Processes, 110(1), 1-22. https://doi.org/10.1016/j.obhdp.2009.05.001

Torralbo, A., Santiago, J., \& Lupiáñez, J. (2006). Flexible conceptual projection of time onto spatial frames of reference. Cognitive Science, 30(4), 745-757. https://doi.org/10.1207/s15516709cog0000_67

Wong, P. T., \& Watt, L. M. (1991). What types of reminiscence are associated with successful aging? Psychology and Aging, 6(2), 272-279. 


\section{Figure Captions}

Figure 1. Past- and future statements ratings among British English and Afrikaners (Experiment 1). Note. Errors bars represent standard error of the mean.

Figure 2. Front-back mappings in British English and Afrikaners (Experiment 1).

Note. Errors bars represent standard error of the mean.

Figure 3. Front-back mappings in different age groups (Experiment 2).

Note. Errors bars represent standard error of the mean.

Figures 4A-C. Front-back mappings under temporal induction (Experiments 3a \& 3b)

Note. Errors bars represent standard error of the mean. 


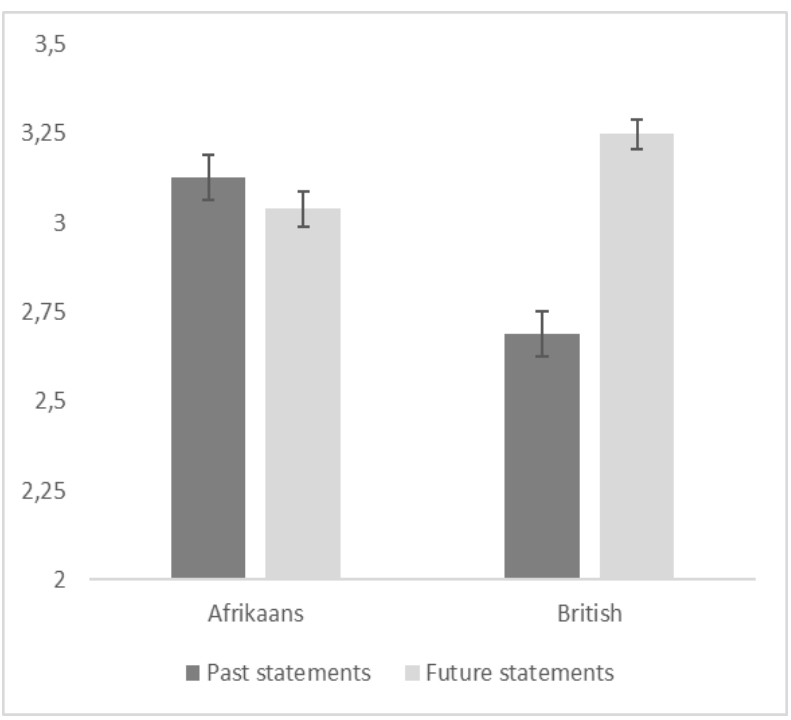




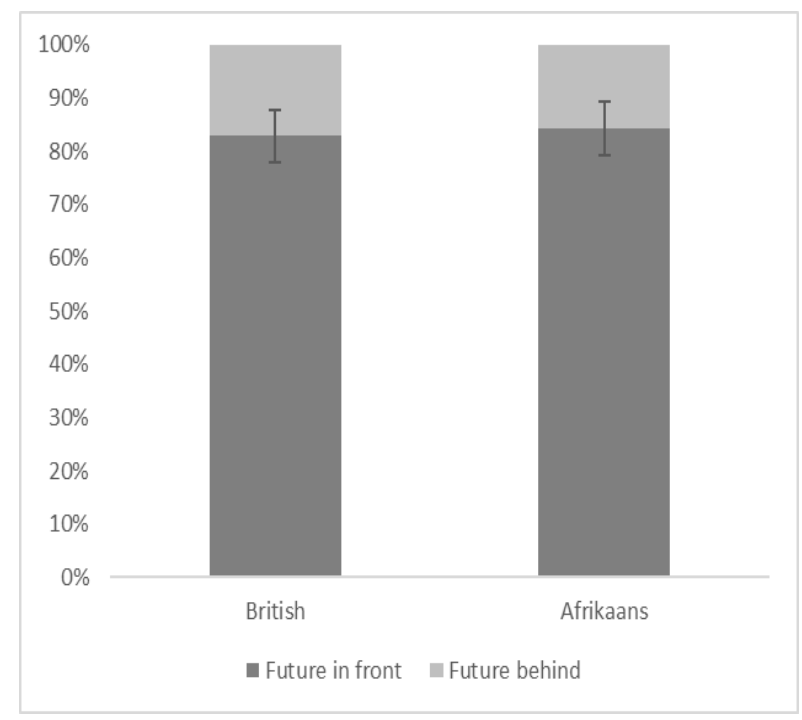




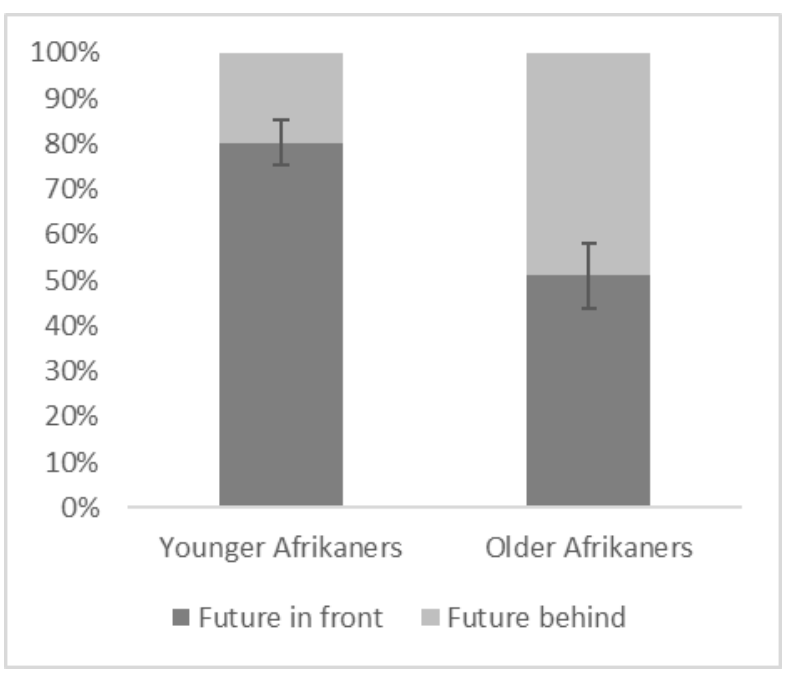




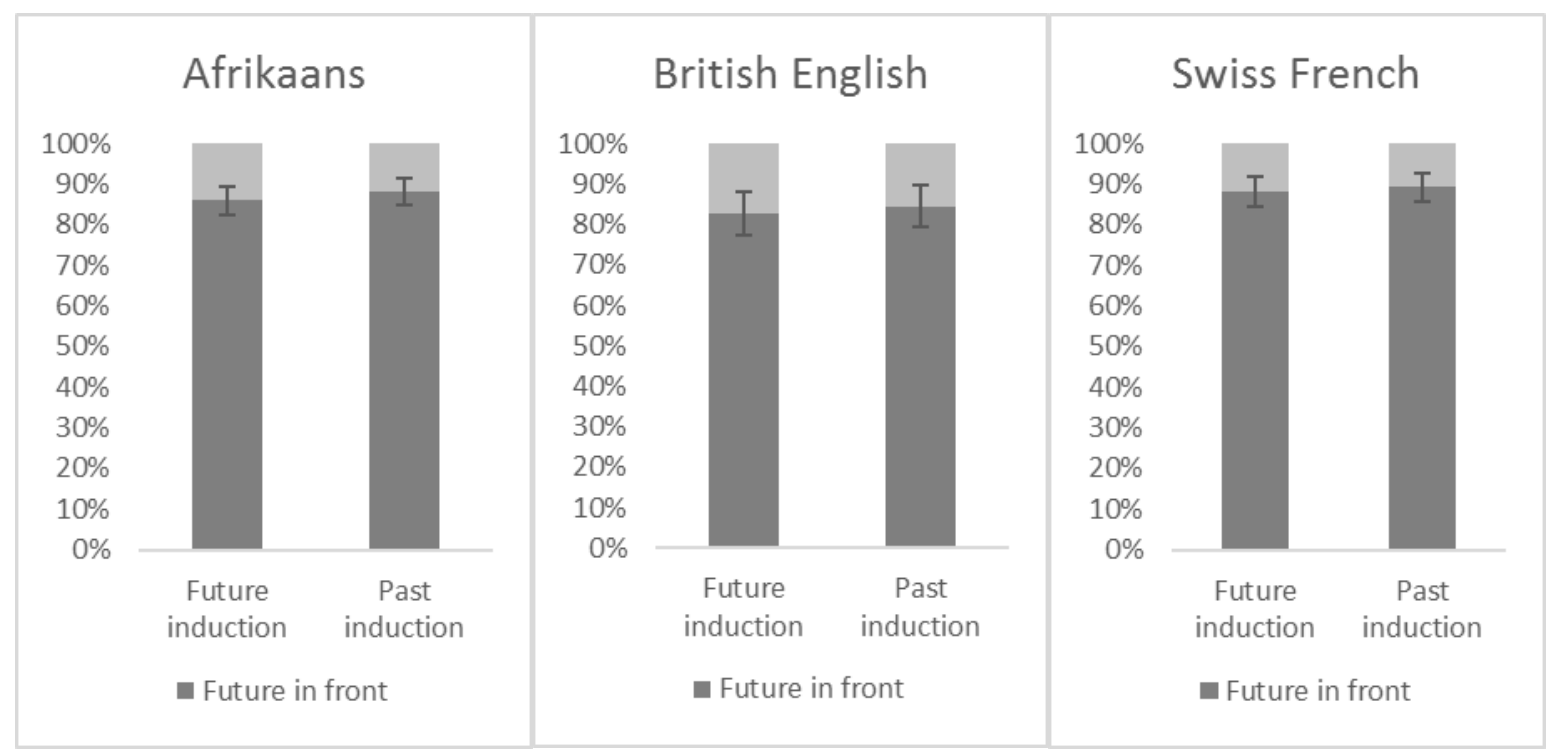

Figure 4A

Figure 4B

Figure 4C 